Ann. Génét. Sél. anim., I977, 9 (3), 283-300.

\title{
Fréquence et pénétrances \\ du gène responsable du Syndrome d'Arthrogrypose et de Palatoschisis dans le bétail charolais en France
}

\author{
G. LEFORT $(*)$, J. J. LAUVERGNE $(* *)$ et P. FABREGUE $(* * *)$ \\ (*) Chaire de Mathématique, Institut national agronomique, I6, rue Cl.-Bernard, \\ 75005 Paris. \\ (**) Département de Génétique animale, \\ Centre national de Recherches zootech»iques I.N.R.A., \\ 78350 Jouy-en-Josas \\ (***) Ingénieur ITEB, Coopérative Agricole Départementale d'élevage, \\ Chalain-le-Comtal, 42210 Montrond-les-Bains.
}

\begin{abstract}
Résumé
Afin d'estimer la fréquence et les pénétrances chez les homozygotes du gène récessif donnant le Syndrome d'Arthrogrypose et de Palatoschisis (SAP) dans la race bovine Charolaise on a utilisé les résultats de xo campagnes annuelles de testage des jeunes mâles en race pure conduites en France depuis 1966-1967. Les équations utilisées étaient celles établies par LAUVERGNE et LEFORT (C.R. Acad. Sci. Paris, I973, série D, 227, 2793-2796). Les calculs ont été faits sur ordinateur, par itération.

Il s'agit d'une analyse des fratries de demi-germains constituées par les 23064 descendants des $\Upsilon_{5} 6$ taurillons testés retenus, après l'exclusion de 17 d'entre eux dont le père avait lui-même été testé (taille moyenne d'un lot de testage : $r_{4} 6$ veaux des deux sexes). On a noté ro3 veaux anormaux $\operatorname{SAP}(0,45 \%$ des naissances) et 43 taureaux se sont révélés transmetteurs de la tare $(27,6 \%$ des taureaux testés). Certains de ces transmetteurs détectés étaient probablement homozygotes porteurs sains mais cette probabilité n'atteint que la valeur 0.66 dans le meilleur des cas. L'estimation de la fréquence du gène dans la population Charolaise française était, avant toute tentative d'éradication, $\widehat{q}=0,197$, avec une intervalle de confiance de 0,13 à 0,26 au seuil de 95 p. Ioo. Les estimations des pénétrances chez les homozygotes mâles et femelles étaient respectivement $\widehat{w^{\prime}}=0,145$ et $\widehat{w^{\prime \prime}}=0,089$ avec des intervalles de confiance de, respectivement, 0,06-0,23 et 0,03-0,15. La pénétrance chez les mâles était significativement supérieure à celle des femelles. La probabilité de non-détection d'un mâle hétérozygote est encore de o, I6 pour des lots de testage de I 50 descendants et celle d'un homozygote porteur sain de 0,025 , dans les conditions qui prévalent actuellement dans le Charolais français.
\end{abstract}




\section{Introduction}

Le Syndrome d'Arthrogrypose et de Palatoschisis (SAP) en race Charolaise est étudié depuis une dizaine d'années, principalement en France et au Canada (cf. LAUVERGNe et FAUCON, I976, pour revue). On admet que la tare est due à un mutant autosomal, récessif à pénétrance incomplète.

L'estimation de la fréquence et des pénétrances peut se faire en utilisant la méthode de Lauvergne et Lefort (I973). C'est ainsi qu'ont déjà été obtenues les valeurs données par LAUVERGNE (I975) mais avec des approximations importantes et sans discussion de la précision. Dans le présent article, nous présentons une étude complète du problème à partir des données de dix campagnes de testage des jeunes taureaux Charolais sur leur descendance, en race pure. La situation étudiée est celle existant jusqu'à une date récente, avant toute tentative systématique d'éradication du gène.

\section{II. - Matériel et méthodes}

\section{A. - Rappel sur les méthodes indirectes d'étude du comportement des gènes à effet visible et de leur tréquence chez les bovins}

Chez les bovins il est généralement difficile de mettre en place une expérimentation pour l'étude directe des facteurs mendéliens à effet visible. On doit avoir recours à des méthodes d'approche indirectes. Le prototype de ces méthodes, mises au point d'abord en génétique humaine, est la méthode dite des "indicateurs " (Proband Methode) proposée déjà par Weinberg (I928) en Allemagne, cf. Huron et RufFí,, (I959) et LI, (I96I) pour revue. Ces méthodes s'attachent à l'étude des fratries signalées par un proposant (ou indicateur) qui porte la tare; elles testent une hypothèse globale : la tare est héréditaire et conditionnée par un gène récessif à pénétrance complète.

En général, chez l'homme, on utilise des fratries de germains (pleins frères ou sœurs). Dans l'espèce bovine, toutefois, les fratries de germains sont rares, on peut alors utiliser les $3 / 4$ de germains (un parent et un grand parent de l'autre côté en commun) comme le montrent Huston et al. (r974) qui ont étudié l'application des méthodes des indicateurs dans cette espèce, ou même les demi-germains, mais alors la connaissance de la fréquence génique dans la population devient nécessaire.

La méthode que nous avons proposée (LAUVERGNE et LEForT, I973) s'applique aux données de demi-germains (en l'occurrence les groupes de descendants de taureaux de testage) mais elle considère le problème d'une manière un peu différente, aboutissant à une estimation de la pénétrance et de la fréquence génique en supposant, au préalable, qu'il s'agit d'un facteur mendélien récessif.

Afin de vérifier les conditions de départ (monofactorialité et récessivité) il a donc fallu imaginer une procédure complémentaire (LAUVERGNE, I974) avec un raisonnement qui a fait appel aux notions de symptomatologie (analyse des différents symptômes pour isoler des syndromes) déjà abordées par RIECK et al (1973). Cependant notre approche a été un peu différente de celle des auteurs allemands : on s'est tout d'abord attaché à démontrer que chaque symptôme, 
pris indépendamment, était dû à un facteur mendélien avant de prouver, par l'absurde, qu'un seul facteur était responsable d'un syndrome regroupant tous les symptômes, dont les plus constants étaient la fissure palatine et les anomalies, des membres. On a ensuite déduit de l'examen de croisements industriels que ce facteur responsable du Syndrome d'Arthrogrypose et de Palatoschisis (SAP) était un récessif.

\section{B. - Les données}

Les fratries de demi-germains parmi lesquelles on a pu compter le nombre des anormaux présentant le syndrome SAP sont constituées par les groupes de descendants des taurillons mis au testage en race Charolaise en France.

Le testage des mâles Charolais sur leur descendance en race pure opère par campagne annuelle dont la première remonte à I966-67, (I966 mise en place des I.A., I967 naissance des veaux). Il a été organisé tout d'abord par l'Association de testage de la race Charolaise (ATRC) et, depuis I972, par des coopératives d'élevage et d'insémination artificielle regroupées dans l'Union de Testage charolaise.

De jeunes mâles, dont le nombre varie entre $\mathrm{r} 2$ et 24 , sont choisis, chaque année dans les élevages Charolais inscrits. La semence de ces mâles est mise en place dès le mois d'avril et jusqu'à juin sur des femelles Charolaise non inscrites de race pure, prises au hasard. Limité les premières années au département de l'Allier, le testage se pratique maintenant sur 9 départements : outre l'Allier, la Vendée, la Loire, la Saône-et-Loire, la Creuse, l'Indre, le Cher, l'Yonne et la Nièvre.

Le nombre des inséminations mises en place correspond à environ 150 naissances par taureau mais ce nombre est soumis à des fluctuations importantes suivant les années et les taureaux du fait de la difficulté de synchroniser en un court laps de temps ( 3 mois) la mise en place de la semence des jeunes mâles sur une zone aussi vaste.

Les éleveurs renvoient en principe les cartes de toutes les naissances, même quand il s'agit de morts-nés ou d'anormaux. Ces derniers sont alors vus par le contrôleur, qui note les tares apparentes.

Les études symptomatologiques et syndromatologiques déjà citées permettent d'identifier à peu près sûrement les animaux présentant le syndrome; ceux qui ont au moins l'un des deux symptomes suivants : fissure palatine ou arthrogrypose des membres sont considérés comme tarés. Les données des dix premières campagnes sont rassemblées dans le tableau $\mathrm{I}$.

On peut considérer que, dans les premières années du testage, les critères du choix des taureaux étaient indépendants de la présence du gène SAP, présence d'ailleurs généralement inconnue, et nous avons admis que les animaux testés formaient un échantillon aléatoire de la population étudiée. Toutefois, approximativement à partir de 1973 (campagnes 8, 9 et Io), dans le contingent d'animaux soumis au testage et issus de pères testés, n'ont été introduits que des fils de taureaux réputés indemnes de la tare. Ce choix systématique fait que ces animaux ne sont pas représentatifs de la population et ne peuvent être utilisés pour l'étude de la population avant toute tentative d'éradication. Ces animaux sont donnés dans le tableau 2.

Le tableau 3 est une récapitulation des données année par année avec, pour les campagnes 8, 9 et Io, des statistiques portant : I) sur l'ensemble des données, 2) sur les données après élimination des descendants d'animaux issus de père testés. 


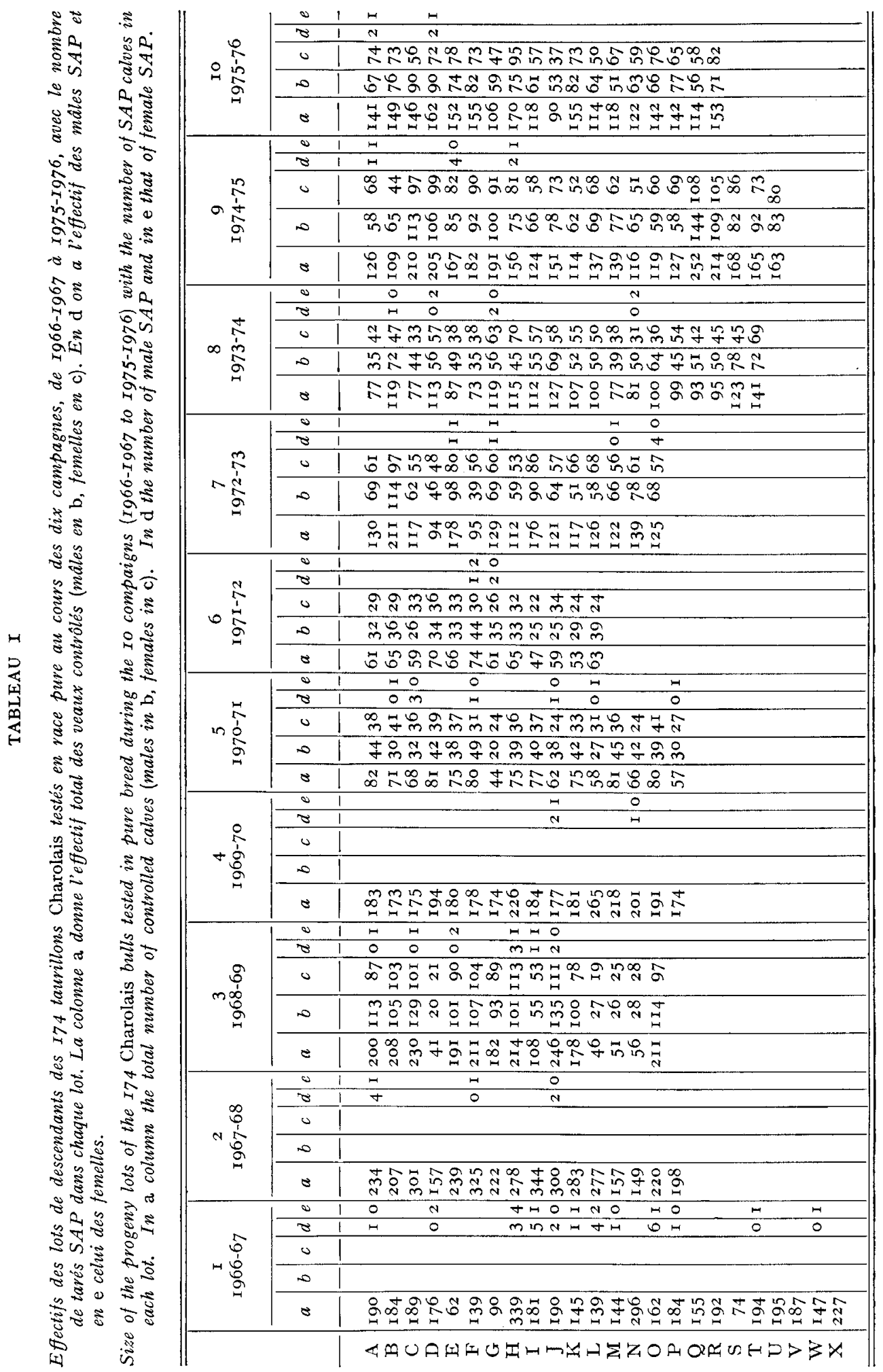


TABLEAU 2

Liste des taureaux Charolais testés en race pure et dont les pères ont déjà été testés

Charolais bulls on the pure breed testing program whose fathers have already been tested.

\begin{tabular}{|c|c|c|c|c|c|c|c|}
\hline \multirow{3}{*}{$\mathrm{No}^{\mathrm{o}}\left({ }^{1}\right)$} & \multicolumn{5}{|c|}{ Nombre de descendants } & \multirow{2}{*}{\multicolumn{2}{|c|}{ Père }} \\
\hline & \multicolumn{3}{|c|}{ Contrôlés } & \multicolumn{2}{|c|}{ SAP } & & \\
\hline & $T$ & M & $\mathrm{F}$ & M & $\mathrm{F}$ & $\mathrm{N}^{\circ}$ & Remarques \\
\hline L8 & roo & 50 & 50 & o & 0 & VI & non $\mathrm{TD}\left({ }^{2}\right)$ \\
\hline Q8 & 100 & 64 & 36 & o & o & $\mathrm{C}_{5}$ & non $T D$ \\
\hline P8 & 99 & 45 & 54 & o & o & $\mathrm{N}_{5}$ & non TD \\
\hline Q8 & 93 & $5^{I}$ & 42 & o & o & RI & non $T D$ \\
\hline$\tilde{\mathrm{T}} 8$ & I $4 \mathrm{I}$ & 72 & 69 & o & o & $M_{4}$ & non $T D$ \\
\hline A9 & 126 & $5^{8}$ & 69 & I & I & $x\left({ }^{3}\right)$ & non TD \\
\hline $\mathrm{C} 9$ & 210 & II 3 & 97 & o & o & $\mathrm{G}_{2}$ & non $T D$ \\
\hline G9 & I9I & 100 & $9 \mathrm{I}$ & o & o & L6 & non $T D$ \\
\hline P9 & 127 & 58 & 69 & o & o & $y\left({ }^{3}\right)$ & non TD \\
\hline R9 & 214 & 109 & 105 & o & o & J3 & non TD \\
\hline S9 & I 68 & 82 & 86 & o & o & $\mathrm{P}_{4}$ & non TD \\
\hline FIo & I 55 & 82 & 73 & o & o & RI & non TD \\
\hline JIo & 90 & 53 & 37 & o & o & $\mathrm{H}_{2}$ & non TD \\
\hline Mio & II 8 & $5 \mathrm{I}$ & 67 & o & o & $\mathrm{G}_{4}$ & non $\mathrm{TD}$ \\
\hline OIo & $I_{42}$ & 66 & 76 & o & 0 & $\mathrm{E} 6$ & non $\mathrm{TD}$ \\
\hline Pro & 142 & 77 & 65 & o & o & E6 & non $T D$ \\
\hline Rro & I 53 & $7 \mathrm{r}$ & 82 & o & o & $\mathrm{G}_{4}$ & non $T D$ \\
\hline
\end{tabular}

(1) Les taureaux sont désignés d'après le no de la campagne, la lettre de leur indicatif est celle de la ligne où ils sont référencés dans le tableau $I$. article.

(2) TD : transmetteur détecté.

$\left.{ }^{3}\right) x$ et $y$ sont des taureaux testés en dehors du programme des ro campagnes analysées dans le présent

Le sex-ratio des animaux tarés s'écarte quelque peu de celui de la population; il faut donc considérer que les pénétrances sont différentes dans les deux sexes. Dans ce cas, les formules utilisées, qui sont rappelées plus loin, nécessitent de connaître le nombre des descendants mâles et femelles dans chaque lot de testage. Quand cette information manquait, (campagnes $I, 2$ et 4) nous avons utilisé les estimations obtenues en multipliant la taille du lot de testage par les proportions des mâles et des femelles dans la population.

\section{C. - Méthodes statistiques}

Ces méthodes sont développées dans l'annexe.

\section{Notations}

$t$ : nombre de taureaux testés

$s_{0}$ : nombre de taureaux reconnus transmetteurs

$n_{j}$ : effectif du lot de "testage " $j$ dont $m_{j}$ mâles et $f_{j}$ femelles

$n_{0}$ : effectif total des veaux tarés dont $m_{0}$ mâles et $f_{0}$ femelles 


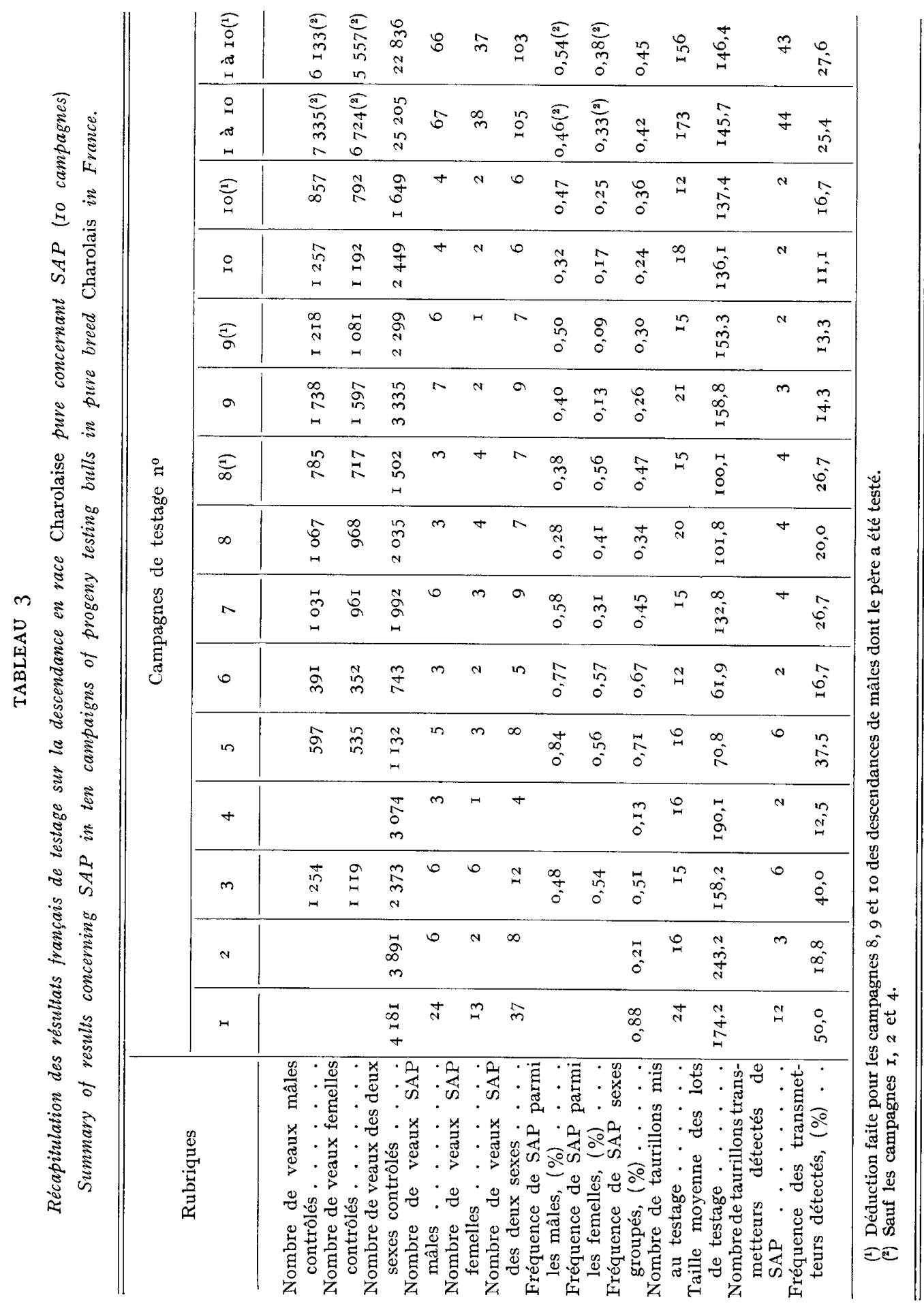


$x_{0}$ : nombre total des veaux tarés dont $x^{\prime}{ }_{0}$ mâles et $x^{\prime \prime}{ }_{0}$ femelles

$q$ : fréquence du gène dans la population (les fréquences chez les mâles et femelles sont supposées suffisamment voisines pour être confondues).

$w^{\prime}:$ pénétrance chez les mâles

$w^{\prime \prime}$ : pénétrance chez les femelles

De manière systématique les minuscules désignent des valeurs numériques (paramètres ou observations) les majuscules désignant les aléatoires correspondantes (estimateurs, lorsqu'il s'agit de paramètres). Par exemple $\mathrm{W}^{\prime}$ est l'estimateur de la pénétrance $w^{\prime}$ chez les mâles; l'estimation correspondante est notée $\widehat{w^{\prime}}$.

2. Définition des estimations de $\mathrm{q}, \mathrm{w}^{\prime}$ et $\mathrm{w}^{\prime \prime}$.

Les estimations sont les solutions du système d'équations II de la note de LAUVERGNE et LEFORT (I973).

(2) $\quad x_{0}^{\prime \prime}=\frac{f_{0} q^{2} w \omega^{\prime \prime}\left(\mathbf{I}-q w^{\prime}\right)}{\mathbf{I}-q^{2} w^{\prime}}$

II

(3) $t-s_{0}=\frac{t}{\mathrm{I}-q^{2} w^{\prime}}$

$\times \sum_{j=1}^{t}\left\{\left[\mathrm{I}-q\left(\mathrm{I}-\exp -\frac{q}{2}\left(m_{j} w^{\prime}+f_{j} w^{\prime \prime}\right)\right)\right]^{2}-q^{2} w^{\prime} \exp -q\left(m_{j} w^{\prime}+f_{j} w^{\prime \prime}\right)\right\}$.

L'équation (3') qui remplace l'équation (3) de II est celle donnée à la fin de la note.

\section{Calcul des estimations}

Il a été fait par itération.

Partant des valeurs $q_{n}, w_{n}^{\prime}$, w ${ }_{n}^{\prime \prime}\left(n=0\right.$, I...) les valeurs suivantes $q_{n+1}, w_{n+1}^{\prime}$ et $w^{\prime \prime}{ }_{n+1}$ sont les solutions du système d'équations.

$(\mathrm{I})_{n}$

$$
\begin{aligned}
& x_{0}^{\prime}=\frac{m_{0} q^{2}{ }_{n} W^{\prime}{ }_{n+1}\left(\mathrm{I}-q_{n} W_{n}^{\prime}\right)}{\mathrm{I}-q_{n} w^{\prime}{ }_{n}} \\
& x_{n}^{\prime \prime}=\frac{f_{0} q_{n} w^{\prime \prime}{ }_{n+\mathbf{1}}\left(\mathrm{I}-q_{n} w_{n}^{\prime}\right)}{\mathrm{I}-q_{n} w^{\prime}{ }_{n}}
\end{aligned}
$$

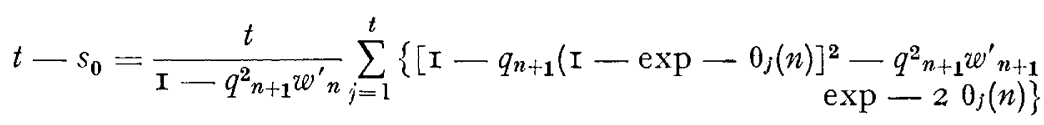

$(2)_{n}$

$\left(3^{\prime}\right)_{n}$

où

$$
\theta_{j}(n)=\frac{I}{2} q_{n}\left(m_{j} w^{\prime}{ }_{n}+f_{j} w_{n}^{\prime \prime}\right)
$$


Les valeurs de $w_{n+1}^{\prime}$ et $w^{\prime \prime}{ }_{n+1}$ s'obtiennent immédiatement dans les équations (I) ${ }_{n}$ et $(2)_{n}$; la valeur de $q_{n+1}$ est la racine positive de l'équation $\left(3^{\prime}\right)_{n}$ qui est du second degré en $q_{n+1}$.

Le calcul a été arrêté quand la valeur absolue de la différence $q_{n+1}-q_{n}$ était inférieure à $\mathrm{IO}^{-3}$.

Les programmes de calcul ont été écrits en FORTRAN et sont disponibles à la Chaire de Mathématiques de l'INA. Ils donnent aussi, pour chaque taureau transmetteur la probabilité qu'il soit homozygote.

4. Variances et covariances des estimateurs

On a déterminé des valeurs approchées valables pour les lois asymptotiques en linéarisant les équations du système précédent.

\section{III. - Résultats}

I) Les estimations de $q$, w' et $w w^{\prime \prime}$ sont :

$\widehat{q}=0$, I97

$\widehat{w}^{\prime}=0, \mathrm{I} 45$

$\widehat{w}^{\prime \prime}=0,089$

2) La matrice des variances et covariances asymptotiques des estimateurs $Q, W^{\prime}$ et $W^{\prime \prime}$ est :

$$
\operatorname{IO}^{-4}\left(\begin{array}{rrr}
\text { IO,O } & -\mathrm{II}, \mathrm{I} & -6,8 \\
-\mathrm{II}, \mathrm{I} & \mathrm{I} 7,7 & 8,9 \\
-6,8 & 8,9 & 7,6
\end{array}\right)
$$

3) Les intervalles de confiance approchés au niveau 95 p. Ioo s'en déduisent immédiatement :

$$
\begin{aligned}
& 0, \text { I3 }<q<0,26 \\
& 0,06<w^{\prime}<0,23 \\
& 0,03<w^{\prime \prime}<0, \text { I5 }
\end{aligned}
$$

4) Probalités d'homozygotie.

Pour les 43 taureaux transmetteurs, les probabilités de l'état homozygote vont de o à 0,67 :

26 sont comprises entre o et $\mathrm{O,I}$

Io sont comprises entre $0, I$ et 0,2

4 sont comprises entre 0,2 et 0,3 .

les trois plus grandes sont égales à 0,$44 ; 0,57$ et 0,67 . 


\section{IV. - Discussion}

\section{A. - Comparaison avec les précédents résultats}

Les précédentes estimations de $\widehat{q}$, $w^{\prime}$ et $w^{\prime \prime}$ sont données dans le tableau no 4 .

\section{TABLEAU 4}

Estimations successives de la fréquence du gène responsable de $S A P$, en race Charolaise en France (q) et des pénétrances chez les homozygotes ( $\mathrm{w}^{\prime}$ et $\mathrm{w}^{\prime \prime}$ ).

Successive estimations of the frequency of the gene for $S A P$ in French Charolais cattle (q) and of penetrances in homozygotes ( $\mathrm{w}^{\prime}$ and $\left.\mathrm{w}^{\prime \prime}\right)$.

\begin{tabular}{|c|c|c|c|c|c|}
\hline \multirow{2}{*}{ Références } & \multicolumn{4}{|c|}{ Estimations de } & \multirow{2}{*}{ Observations } \\
\hline & $q$ & $w^{\prime}$ & $w^{\prime \prime}$ & $w$ & \\
\hline 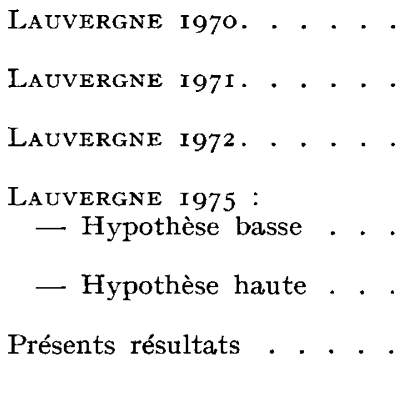 & $\begin{array}{l}0,15 \\
0,18 \\
0,20 \\
0,200 \\
0,202 \\
0,197\end{array}$ & 0,147 & 0,089 & $\begin{array}{l}0,115 \\
0, I_{3} \mathrm{I}\end{array}$ & 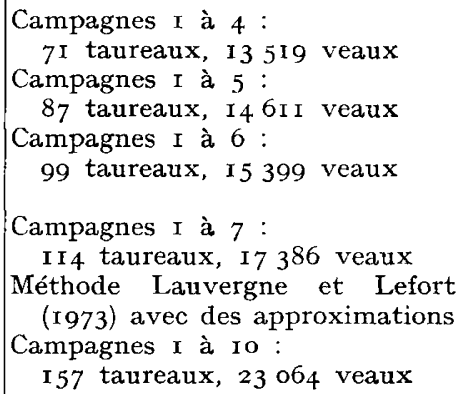 \\
\hline
\end{tabular}

Les valeurs que nous calculons sont finalement assez voisines des estimations obtenues précédemment sur un nombre plus restreint de données par des méthodes approchées.

\section{B. - Précision des estimations}

Ces estimations et surtout celles de $w^{\prime}$ et $w^{\prime \prime}$ sont assez imprécises, comme le montre la longueur des intervalles de confiance.

La plus grande part de l'information utilisée est constituée par les nombres des mâles et des femelles tarés dans les descendances des taureaux testés. Les lois de ces aléatoires dépendent des deux paramètres $q w^{\prime}$ et $q w^{\prime \prime}$; d'où deux conséquences :

- Les variabilités relatives de $Q W^{\prime}$ et $Q W^{\prime \prime}$ sont voisines et inférieures de près de moitié à celles de $\mathrm{W}^{\prime}$ et $\mathrm{W}^{\prime \prime}$;

- Les produits $Q W^{\prime}$ et $Q W^{\prime \prime}$ étant peu variables, les variations de $Q$ et $W^{\prime}$ d'une part, $Q$ et $W^{\prime \prime}$ d'autre part sont liées et de sens contraire, ce qui se traduit par de fortes corrélations négatives entre $Q$ et $W^{\prime}$ et $Q$ et $W^{\prime \prime}(-0,83$ et - 0,78$)$. 


\section{C. - Différence de pénétrance dans les deux sexes}

Les résultats obtenus confirment que les pénétrances sont différentes chez les mâles et les femelles : en effet la différence des estimations de $\mathrm{W}^{\prime}$ et $\mathrm{W}^{\prime \prime}$ a une variance :

$$
v=\operatorname{var} \mathrm{W}^{\prime}+\operatorname{var} \mathrm{W}^{\prime \prime}-2 \operatorname{cov}\left(\mathrm{W}^{\prime}, \mathrm{W}^{\prime \prime}\right)=10^{-4} \times 7,5
$$

ce qui implique :

$$
\frac{\widehat{w}^{\prime}-\widehat{w}^{\prime \prime}}{\sqrt{v}}=2,04
$$

En utilisant la normalité asymptotique des estimations $\mathrm{W}^{\prime}$ et $\mathrm{W}^{\prime \prime}$, on peut donc affirmer que la différence $\mathrm{W}^{\prime}-\mathrm{W}^{\prime \prime}$ est significativement différente de $o$ au niveau de 5 p. Ioo (en effet, la valeur prise par la variable réduite : $\left(w^{\prime}=w^{\prime \prime}\right) / \sqrt{v}$ dépasse $I, 96)$.

\section{D. - Existence de taureaux homozygotes}

Les probabilités a posteriori de l'état homozygote pour les 43 taureaux transmetteurs montrent qu'il existe très probablement des homozygotes dans l'échantillon des taureaux testés (l'espérance du nombre de ces homozygotes est de l'ordre de 4), mais il n'est pas possible d'identifier ces homozygotes puisque, dans le meilleur des cas, il y a seulement 2 chances sur 3 que le taureau soit homozygote. Ces résultats sont en accord avec ceux de Goonewardene et Berg (I976) qui, au Canada, ont pu identifier des transmetteurs homozygotes porteurs sains comme le laisse prévoir la valeur incomplète de la pénétrance.

\section{E. - Probabilité de non détection d'un mâle transmetteur}

Les faibles valeurs obtenues pour les pénétrances et la taille habituelle des lots de testage font qu'une proportion importante de taureaux transmetteurs échappent à la détection (ce que nous avons déjà souligné, LAUVERGNE, I975).

La probabilité de non détection d'un transmetteur est la probabilité que, dans sa descendance il n'y ait aucun mâle taré et aucune femelle tarée soit; $m$ étant le nombre de mâles et $f$ le nombre de femelles du lot de testage :

$$
\exp -q \frac{m w^{\prime}}{2} \times \exp -q \frac{f w^{\prime \prime}}{2}=\exp -q \frac{m w^{\prime}+f w^{\prime \prime}}{2}
$$

pour un hétérozygote, et $\exp -q\left(m w^{\prime}+f w^{\prime \prime}\right)$ pour un homozygote récessif.

Les courbes de la figure I donnent les valeurs de ces probabilités en fonction de la taille du lot de testage (il a été admis que la proportion de mâles et de femelles dans le lot de testage était la même que dans la population). Les valeurs choisies pour $q$, w' et w" sont respectivement 0,$2 ; 0,15$ et 0,09, c'est-à-dire les valeurs estimées dans la population.

Remarquons que, si la taille du lot de testage est égale à cent, la probabilité de non-détection atteint 0,3 pour un hétérozygote et qu'elle est encore de $0, \mathrm{I}$ pour un lot de taille deux cents. 
F. - Information apportée par les taureaux fils de taureaux testés éliminés lors de l'estimation de $\mathrm{q}, \mathrm{w}^{\prime}$ et $\mathrm{w}^{\prime \prime}$

Ces I7 taureaux n'ont pas été pris en compte parce qu'ils ont été choisis systématiquement parmi les descendants de taureaux non reconnus transmetteurs et ne sont donc pas représentatifs de la population. Mais, ce faisant, nous négligeons l'information qu'ils pouvaient apporter et, bien que cette information soit assez pauvre, il est intéressant de vérifier si ces nouvèlles données sont en accord avec les résultats de l'étude précédente.

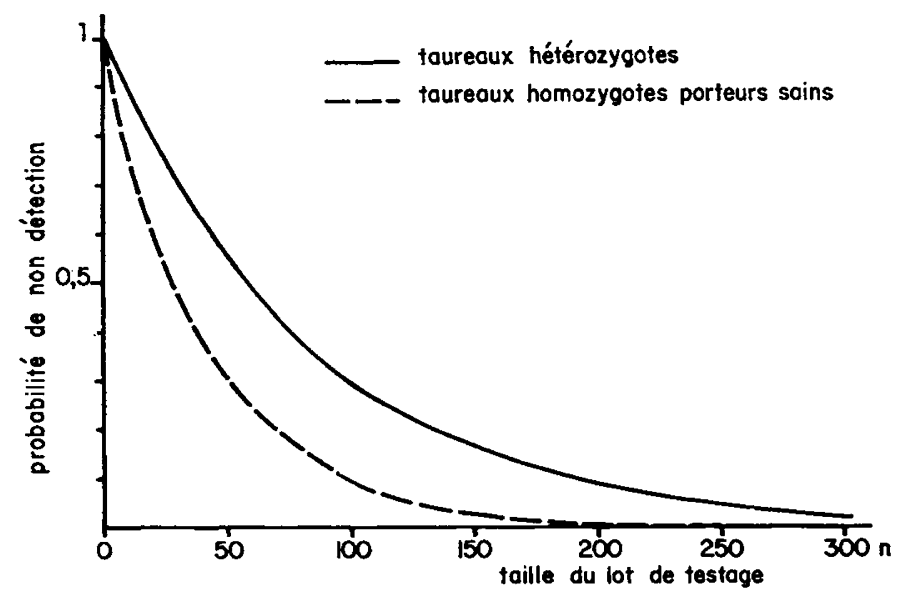

FIG. I. - Probabilité de non détection de taureaux Charolais hétérozygotes et homozygotes porteurs sains du gène récessif responsable de SAP en fonction de la taille des lots de testage (condition du Charolais français avant toute tentative d'éradication).

Probability of non detection of heterozygous and homozygous normal overlap Charolais bulls wearing the recessive gene for $S A P$ according to the size of the progeny lot (french Charolais conditions before any attempt of evadication).

I) Pour un taureau testé et non reconnu transmetteur, on peut calculer les probabilités a posteriori des trois génotypes (l'étude est analogue à celle présentée en IV dans l'annexe) et, à partir de là, la probabilité qu'un fils de ce taureau soit reconnu transmetteur. On en déduit aisément la loi de l'aléatoire Y égale au nombre de transmetteurs reconnus parmi les I7 fils de taureaux testés (les paramètres $q, w, w^{\prime}$ sont remplacés par leurs estimations) :

- l'espérance de $Y$ est légèremant supérieure à 3;

- la probabilité que Y soit égal à o ou I est de l'ordre de $0, \mathrm{I} 7$. Le résultat expérimental $(y=I)$ est faible, mais ce n'est pas en contradiction avec l'étude antérieure, puisque la probabilité d’obtenir un résultat aussi faible est de o,I7.

2) Dans la descendance du seul taureau reconnu transmetteur (58 mâles et 68 femelles), on a trouvé un mâle taré et une femelle tarée. Les aléatoires $\mathrm{X}^{\prime}$ et $\mathrm{X}^{\prime \prime}$ relatives à ce taureau ont des lois de Poisson de paramètres 0,84 et 0,6 I si le taureau est hétérozygote hypothèse la plus vraisemblable, I,68 et I,22 si le taureau est 
homozygote, récessif. Les valeurs expérimentales $x^{\prime}=\mathrm{I}$ et $x^{\prime \prime}=\mathrm{I}$ sont, pour ces lois, des valeurs très vraisemblables; elles sont en excellent accord avec l'étude antérieure.

Reçu pour publication en juillet 1977

\section{Summary}

\section{Frequency an penetrances of the gene for the syndrome of arthrogyposis and palatoschisis in the French Charolais cattle}

To estimate the frequency and penetrance of the recessive gene for the Syndrome of Arthrogyposis and Palatoschisis (SAP) in homozygotes of the Charolais breed, we used the data obtained from Io years of testing young pure-bred males in France, since 1966-1967. The equations applied were established by LAuvergne and LeFort (C.R. Acad. Sci. Paris, 1973, série D, 227, 2 793-2 796); computation was done on a computer by iteration.

Half sibships constituted of 23064 progeny of 157 bull-calves tested were analyzed; the sire of 17 of these having himself been tested, they were eliminated. The mean size of a testing lot was I 46 calves of both sexes. There were I03 abnormal SAP calves ( $0.45 \%$ of births) and 43 bulls were proven to transmit the defect $(28 \%$ of bulls tested). Some of these detected transmitters were certainly normal overlap homozygous carriers, but this could only be affirmed with 0.66 probability in the best case. Gene frequency estimation in the French Charolais population before an attempt at eradication was $\widehat{q}=0.197$, with a confidence interval of $0.13-0.26$ at a 95 p. Ioo threshold. Penetrance estimations in male and female homozygotes were $\widehat{w^{\prime}}=0.145$ and $\widehat{w^{\prime \prime}}=0.089$, respectively, with confidence intervals of $0.06-0.23$ and $0.03-0.15$ respectively. Penetrance in males was significantly higher than that of females. The non-detection probability of a heterozygous male is still 0.16 for testing lots of $\times 5^{\circ}$ progeny and that of a healthy homozygous. carrier 0.025 in present conditions of the French Charolais.

\section{Références bibliographiques}

Goonewardene L. A., Berg R. T., 1976. Arthrogryposis in Charoiais cattle, a study on gene penetrance. Ann. Génét. Sél. anim., 8, 493-499.

Huron R., Ruffié J., I959. Les méthodes en Génétique Générale et en Génétique Humaine. Masson, Paris, 449-459.

Huston K., Chase R., Waller R., r974. Identifying genes with visible effects in cattle:full, three quarter and half-sib, other methods. Irst Congr. Genet. appl. anim. Breeding, Madrid, 1, $39-46$.

LAUVERGNe J. J., 1970. Quelques considérations sur la fissure palatine en race Charolaise. In J.M.D., Réunion du 9 juin 1970 du groupe d'Etude du "Caractère Palais fendu en Charolais" ITEB, Section Amélioration Génétique, Paris, pp $6+2$, ronéoté, (suppl. à) (cf. résumé in LAUVERGNE et FAUCON, 1976).

Lauvergne J.J. ,r97I. Déterminisme héréditaire du Syndrome et conséquences pour la sélection du Charolais, in Réunion du groupe d'Étude Palais fendu en Charolais du 13 octobre 1971, ITEB, Paris, pp 2, ronéoté. (cf. résumé in Lauvergne et Faucon, 1976).

Lauvergne J. J., 1972. Hereditary determinism of Arthrogryposis with Palatoschis Syndrome in Charolais cattle. Conférence du 20-6-72 au "Beef Cattle Research Ranch ", de 1'Université d'Alberta à Kinsella, Alberta, non publié. (Cf. résumé in LaUvergne et Faucon, 1976).

LAUvergne J. J., I974. Étude des gènes à effets visibles à partir des données de testage des taureaux sur leur descendance. $\mathrm{I}^{\mathrm{er}}$ Congr. Mond. Génét. appl. élevage, Madrid, 1, 47-55.

Lauvergne J. J., r975. État actuel des connaissances sur le Syndrome d'Arthrogrypose et de Palatoschisis (SAP) dans le bétail Charolais de France. Ann. Génét. Sél. anim., 7, 321-33o.

Lauvergne J. J., Faucon A., 1976. Le Syndrome d'Arthrogrypose et de Palatoschisis (SAP) en race bovine Charolaise : bibliographie annotée, 1967-1975. Ann. Génét. Sél. anim., 8, 51-70. 
LAuvergne J. J., Lefort G., I 973. Nouvelle méthode pour analyser le comportement et la fréquence des gènes récessifs à effets visibles dans les populations bovines. C.R. Acad. sci Paris (Série D), 227, 2 793-2 796.

Lefort G., Ollivier L., Sellier P., I975. - Analyse du comportement et de la fréquence des gènes à effets visibles dans les fraties de germains et de demi-germains. Ann. Génét. Sél. anim., 7, $365-377$.

LI, C. C., I96r. Human Genetics. Mc Graw Hill, New-York, 58-78.

Rieck G. W., Herzog A., RaU W., I973. Untersuchungen zur teratologischen Populationsstatistik und zur Ätiologie der embryonalen Entroicklt ngsstörungen beim Rind. II. Missbildungssyndrome. Giessener Beitr. Erbpath. Zuchthyg. 5, (1/2), I-70.

Weinberg W., I928. Mathematische Grundlagen der Probendenmethode. Z. Indukt. Abstam. Vererbungsl., 48, 179-228.

\section{Annexe \\ Étude mathématique du modèle utilisé}

\section{I. - Méthode}

I. Modèle

Le modèle a été défini dans la note de LAuvergne et Lefort (I973).

Les lois des aléatoires $\mathrm{X}_{j}^{\prime}$ et $\mathrm{X}^{\prime \prime}{ }_{j}$ (nombres de tarés mâles et femelles dans le lot de testage $j$ qui comporte $m_{j}$ mâles et $f_{j}$ femelles) et de l'indicatrice $\mathrm{S}_{j}$ (égale à I si le taureau $j$ est reconnu transmetteur et à o sinon) sont définies par :

- les triplets $\mathrm{X}_{j}^{\prime}, \mathrm{X}^{\prime \prime}{ }_{j}, \mathrm{~S}_{j}$ sont indépendants (ce qui suppose l'absence de relations de parenté connue entre les animaux testés);

- les probabilités qu'un taureau soit homozygote récessif, hétérozygote ou non transmetteur sont respectivement :

$$
\frac{q^{2}\left(\mathrm{I}-w^{\prime}\right)}{\mathrm{I}-q^{2} w^{\prime}} \quad \frac{2 q(\mathrm{I}-q)}{\mathrm{I}-q^{2} w^{\prime}} \quad \frac{(\mathrm{I}-q)^{2}}{\mathrm{I}-q^{2} w^{\prime}}
$$

$w^{\prime}$ et $w^{\prime \prime}$ étant les pénétrances du gène chez les homozygotes récessifs respectivement mâles et femelles;

- les lois conditionnelles de $\mathrm{X}_{j}{ }_{j}$ et $\mathrm{X}^{\prime \prime}{ }_{j}$ connaissant le génotype du taureau $j$ sont poissonniennes de paramètres $m_{j} w^{\prime} q$ et $f_{j} w^{\prime \prime} q$ pour un homozygote récessif, $m_{j} w " q / 2$ et $f_{j} w " q / 2$ pour un hétérozygote et o pour un non transmetteur.

\section{Définition des estimateurs.}

La technique est analogue à celle utilisée pour l'étude des fratries de germains et demi-germains (LEFORT et al. I975). 
Les estimateurs $\mathrm{Q}, \mathrm{W}^{\prime}, \mathrm{W}^{\prime \prime}$ des paramètres $q, w^{\prime}, w^{\prime \prime}$ sont obtenus en prenant chacune des aléatoires

$$
\mathrm{X}_{0}^{\prime}=\sum_{j=1}^{t} \mathrm{X}_{j}^{\prime} \quad \mathrm{X}_{0}^{\prime \prime}=\sum_{j=1}^{t} \mathrm{X}_{j}^{\prime \prime} \quad t-\mathrm{S}_{0}=t-\sum_{j=1}^{t} \mathrm{~S}_{j}
$$

comme estimateur de son espérance : $\mathrm{Q}, \mathrm{W}^{\prime}, \mathrm{W}^{\prime \prime}$ sont les solutions du système

$$
\mathrm{X}_{\mathbf{0}}^{\prime}=a\left(\mathrm{Q}, \mathrm{W}^{\prime}, \mathrm{W}^{\prime \prime}\right) \quad \mathrm{X}_{0}^{\prime \prime}=b\left(\mathrm{Q}, \mathrm{W}^{\prime}, \mathrm{W}^{\prime \prime}\right) \quad t-\mathrm{S}_{0}=c\left(\mathrm{Q}, \mathrm{W}^{\prime}, \mathrm{W}^{\prime \prime}\right)
$$

où les fonctions $a, b, c$ sont définies par :

$$
\mathrm{E}\left(\mathrm{X}_{0}^{\prime}\right)=a\left(q, w^{\prime}, w^{\prime \prime}\right) \quad \mathrm{E}\left(\mathrm{X}_{0}^{\prime \prime}\right)=b\left(q, w^{\prime} w^{\prime \prime}\right) \quad \mathrm{E}\left(t-\mathrm{S}_{0}\right)=c\left(q, w^{\prime}, w^{\prime \prime}\right)
$$

Les estimations $\widehat{q}, \widehat{w}^{\prime}, \widehat{w}^{\prime \prime}$ sont donc solutions du système :

$$
x_{0}^{\prime}=a\left(\widehat{q}, \widehat{w}^{\prime}, \widehat{w}^{\prime \prime}\right) \quad x_{0}=b\left(\widehat{q}, \widehat{w}^{\prime}, \widehat{w}^{\prime \prime}\right) \quad t-s_{0}=c\left(\widehat{q}, \widehat{w}^{\prime}, \widehat{w}^{\prime \prime}\right)
$$

qui est résolu par itération.

(Les minuscules $x_{0}^{\prime}, x_{0}^{\prime \prime}, s_{0}$ désignent les valeurs observées).

\section{Loi des estimateurs}

Pour de grandes valeurs du nombre $t$ de taureaux testés (dans le cas étudié $t=\mathrm{I} 54$ ), la loi exacte de $\mathrm{Q}, \mathrm{W}^{\prime}, \mathrm{W}^{\prime \prime}$ peut être remplacée par la loi asymptotique qui est une loi normale et est donc définie par les espérances et les variances et covariances de $Q, W^{\prime}, W^{\prime \prime}$.

- Les estimateurs $Q, W^{\prime}, W^{\prime \prime}$ ont des espérances asymptotiques égales aux paramètres $q, w^{\prime}, w^{\prime \prime}$; ils sont asymptotiquement bien centrés.

- Les variances et covariances asymptotiques s'obtiennent en remplaçant les fonctions $a, b, c$ par leurs différentielles au point $\Pi=\left(q, w^{\prime}, w^{\prime \prime}\right)$, c'est-à-dire en écrivant :

$$
\begin{aligned}
\mathrm{X}_{0}^{\prime}-a(\Pi) & =\frac{\partial a}{\partial q}(\Pi)(\mathrm{Q}-q)+\frac{\partial a}{\partial w^{\prime}}(\Pi)\left(\mathrm{W}^{\prime}-w^{\prime}\right)+\frac{\partial a}{\partial w^{\prime \prime}}(\Pi)\left(\mathrm{W}^{\prime \prime}-w^{\prime \prime}\right) \\
\mathrm{X}^{\prime \prime}{ }_{0}-b(\Pi) & =\frac{\partial b}{\partial q}(\Pi)(\mathrm{Q}-q)+\frac{\partial b}{\partial w^{\prime}}(\Pi)\left(\mathrm{W}^{\prime}-w^{\prime}\right)+\frac{\partial b}{\partial w^{\prime \prime}}(\Pi)\left(\mathrm{W}^{\prime \prime}-w^{\prime \prime}\right) \\
t-\mathrm{S}_{0}-c(\Pi) & =\frac{\partial c}{\partial q}(\Pi)(\mathrm{Q}-q)+\frac{\partial c}{\partial w^{\prime}}(\Pi)\left(\mathrm{W}^{\prime}-w^{\prime}\right)+\frac{\partial c}{\partial w^{\prime \prime}}(\Pi)\left(\mathrm{W}^{\prime \prime}-w^{\prime \prime}\right)
\end{aligned}
$$

La matrice $U$ des variances et covariances des estimateurs $Q, W^{\prime}, W^{\prime \prime}$ est donc définie par :

$$
\mathrm{U}=\mathrm{D}^{-1} \mathrm{~V}^{t} \mathrm{D}^{-1}
$$

où $\mathrm{V}$ est la matrice des variances et covariances des aléatoires $\mathrm{X}_{0}^{\prime}, \mathrm{X}^{\prime \prime}{ }_{0}, t-\mathrm{S}_{0}$ et $\mathrm{D}$ la matrice des dérivées partielles des fonctions $a, b, c$ au point $\Pi=\left(q, w^{\prime}, w^{\prime \prime}\right)$.

Les valeurs exactes $q, w^{\prime}, w^{\prime \prime}$ étant inconnues, on obtient une valeur approchée de $\mathrm{U}$, soit $\widehat{\mathrm{U}}$ en remplaçant $q$, w' et $w^{\prime \prime}$ par leurs estimations $\widehat{q}, \widehat{w}^{\prime}, \widehat{w}^{\prime \prime}$.

- Cette loi normale approchée a été utilisée pour déterminer les intervalles de confiance de $q, w^{\prime}$ et $w^{\prime \prime}$ indiqués dans l'article et faire le test de l'hypothèse $w^{\prime}=w^{\prime \prime}$. 
II. - Calcul des moments d'ordre $I$ et 2 de $\mathrm{X}^{\prime}, \mathrm{X}^{\prime \prime}{ }_{0}, \mathrm{~S}_{\mathbf{0}}$

\section{Calcul des espérances}

L'espérance des aléatoires $\mathrm{X}_{j}{ }_{j}, \mathrm{X}^{\prime \prime}{ }_{j}, \mathrm{~S}_{j}$ s'obtient en pondérant les espérances conditionnelles sachant le génotype du taureau $j$ par les probabilités de ces divers génotypes :

$$
\begin{aligned}
& \mathrm{E}\left(\mathrm{X}_{j}^{\prime}\right)=\frac{2 q(\mathrm{I}-q)}{\mathrm{I}-q^{2} w^{\prime}} \times \frac{m_{j} q w^{\prime}}{2}+\frac{q^{2}\left(\mathrm{I}-w^{\prime}\right)}{\mathrm{I}-q^{2} w^{\prime}} \times m_{j} q w^{\prime}=m_{j} w^{\prime} \mathrm{G}\left(q, w^{\prime}\right) \\
& \mathrm{E}\left(\mathrm{X}_{j}^{\prime \prime}\right)=\frac{2 q(\mathrm{I}-q)}{\mathrm{I}-q^{2} w^{\prime}} \times \frac{f_{j} q w^{\prime \prime}}{2}+\frac{q^{2}\left(\mathrm{I}-w^{\prime}\right)}{\mathrm{I}-q^{2} w^{\prime}} \times f_{j} q w^{\prime \prime}=f_{j} w^{\prime \prime} \mathrm{G}\left(q, w^{\prime}\right)
\end{aligned}
$$

où

$\mathrm{E}\left(\mathrm{I}-\mathrm{S}_{j}\right)=\operatorname{Prob}\left(\mathrm{S}_{j}=0\right)=$

$$
\mathrm{G}\left(q, w^{\prime}\right)=\frac{q^{2}\left(\mathrm{I}-q w^{\prime}\right)}{\mathrm{I}-q^{2} w^{\prime}}
$$

$$
\begin{aligned}
\frac{(\mathrm{I}-q)^{2}}{\mathrm{I}-q^{2} w^{\prime}} \times \mathrm{I}+\frac{2 q(\mathrm{I}-q)}{\mathrm{I}-q^{2} w^{\prime}} \times \exp \left[-\frac{q}{2}\left(m_{j} w^{\prime}+f_{j} w^{\prime \prime}\right)\right]+\frac{q^{2}\left(\mathrm{I}-w^{\prime}\right)}{\mathrm{I}-q^{2} w^{\prime \prime}} \\
\times \exp \left[-q\left(m_{j} w^{\prime}+f_{j} w^{\prime \prime}\right)\right]=\frac{\mathrm{I}}{\mathrm{I}-q^{2} w^{\prime}} \mathrm{H}_{j}\left(q, w^{\prime}, 0_{j}\right)
\end{aligned}
$$

où

$$
\theta_{j}=\frac{q}{2}\left(m_{j} w^{\prime}+f_{j} w\right)
$$

$\mathrm{H}_{j}\left(q, w^{\prime}, \theta_{j}\right)=\left[\mathbf{I}-q+q \exp \left(-\theta_{j}\right)\right]^{2}-q^{2} w^{\prime} \exp \left(-2 \theta_{j}\right)$

En sommant les résultats précédents, on obtient :

$$
\begin{aligned}
& \mathrm{E}\left(\mathrm{X}_{0}^{\prime}\right)=m_{0} w^{\prime} \mathrm{G}\left(q, w^{\prime}\right)=m_{0} w^{\prime} \frac{q^{2}\left(\mathrm{I}-q w^{\prime}\right)}{\mathrm{I}-q^{2} w^{\prime}}=a\left(q, w^{\prime}, w^{\prime \prime}\right) \\
& \mathrm{E}\left(\mathrm{X}_{0}^{\prime \prime}\right)=f_{0} w^{\prime \prime} \mathrm{G}\left(q, w^{\prime}\right)=f_{0} w^{\prime \prime} \frac{q^{2}\left(\mathrm{I}-q w^{\prime}\right)}{\mathrm{I}-q^{2} w^{\prime}}=b\left(q, w^{\prime}, w^{\prime \prime}\right) \\
& \mathrm{E}\left(t-\mathrm{S}_{0}\right)=\sum_{j=1}^{t} \mathrm{H}_{j}\left(q, w^{\prime}, \theta_{j}\right) /\left(\mathrm{I}-q^{2} w^{\prime}\right)=c\left(q, w^{\prime}, w^{\prime \prime}\right)
\end{aligned}
$$

2. Calcul des variances et covariances

- Pour $\mathrm{X}_{j}{ }_{j}$ et $\mathrm{X}^{\prime \prime}{ }_{j}$, nous utiliserons les variances et covariances conditionnelles: sachant le génotype du taureau $j, \mathrm{X}_{j}^{\prime}$ et $\mathrm{X}_{j}{ }_{j}$ sont indépendantes et ont des lois de Poisson de paramètres connus. Nous écrirons ensuite les formules classiques :

$$
\begin{aligned}
& \operatorname{var} \mathrm{X}_{j}^{\prime}=\mathrm{E}\left[\operatorname{var}_{g} \mathrm{X}_{j}\right]+\operatorname{var}\left[\mathrm{E}_{g}\left(\mathrm{X}_{j}^{\prime}\right)\right] \\
& \operatorname{cov}\left(\mathrm{X}_{j}^{\prime}, \mathrm{X}_{j}^{\prime \prime}\right)=\mathrm{E}\left[\operatorname{cov}_{g}\left(\mathrm{X}_{j}^{\prime}, \mathrm{X}_{j}^{\prime \prime}\right)\right]+\operatorname{cov}\left[\mathrm{E}_{g}\left(\mathrm{X}_{j}^{\prime}\right), \mathrm{E}_{g}\left(\mathrm{X}_{j}^{\prime \prime}\right)\right]
\end{aligned}
$$


(l'indice $g$ signifie que l'étude est faite pour la loi conditionnelle connaissant le génotype du taureau $j$; les espérances et covariances sont obtenues en attribuant à chaque génotype la probabilité qui lui correspond dans le modèle).

La variance d'une loi de Poisson est égale à son espérance; on obtient donc :

$$
\begin{aligned}
\mathrm{E}\left[\operatorname{var}_{g} \mathrm{X}_{j}^{\prime}\right]= & \mathrm{E}\left[\mathrm{E}_{g} \mathrm{X}_{j}^{\prime}\right]=\mathrm{E}\left(\mathrm{X}_{j}^{\prime}\right)=m_{j} w^{\prime} \frac{q^{2}\left(\mathrm{I}-q w^{\prime}\right)}{\mathrm{I}-q^{2} w^{\prime}} \\
\operatorname{var}\left[\mathrm{E}_{g}\left(\mathrm{X}_{j}^{\prime}\right)\right] & =\mathrm{E}\left\{\left[\mathrm{E}_{g}\left(\mathrm{X}_{j}^{\prime}\right)\right]^{2}\right\}-\left\{\mathrm{E}\left[\mathrm{E}_{g}\left(\mathrm{X}_{j}{ }_{j}\right)\right]\right\}^{2}=\mathrm{E}\left\{\left[\mathrm{E}_{g}\left(\mathrm{X}_{j}^{\prime}\right)\right]^{2}\right\}-\left\{\mathrm{E}\left(\mathrm{X}_{j}\right)\right\}^{2} \\
& =\frac{2 q(\mathrm{I}-q)}{\mathrm{I}-q^{2} w^{\prime}}\left(\frac{m_{j} q w^{\prime}}{2}\right)^{2}+\frac{q^{2}\left(\mathrm{I}-w^{\prime}\right)}{\mathrm{I}-q^{2} w^{\prime}}\left(m_{j} q w^{\prime}\right)^{2}-\left[m_{j} w^{\prime} \frac{q^{2}\left(\mathrm{I}-q w^{\prime}\right)}{\mathrm{I}-q^{2} w^{\prime}}\right]^{2} \\
& =m^{2}{ }_{j} q^{3} w^{\prime 2}(\mathrm{I}-q)\left[\mathrm{I}-q w^{\prime}(2-q)\right] / 2\left(\mathrm{I}-q^{2} w^{\prime}\right)^{2}
\end{aligned}
$$

et, en faisant la somme de ces deux termes, la variance de $\mathrm{X}_{j}$.

De même :

$$
\begin{aligned}
& \operatorname{var} \mathrm{X}^{\prime \prime}{ }_{j}=\mathrm{E}\left(\mathrm{X}^{\prime \prime}{ }_{j}\right)+f^{2}{ }_{j} q^{3} w^{\prime \prime}(\mathrm{I}-q)\left[\mathrm{I}-q w^{\prime}(2-q)\right] / 2\left(\mathrm{I}-q^{2} w^{\prime}\right)^{2} \\
& \operatorname{cov}\left(\mathrm{X}^{\prime}{ }_{j}, \mathrm{X}^{\prime \prime}{ }_{j}\right)=f_{j} m_{j} q^{3} w^{\prime} w^{\prime \prime}(\mathrm{I}-q)\left[\mathrm{I}-q w^{\prime}(2-q)\right] / 2\left(\mathrm{I}-q^{2} w^{\prime}\right)^{2}
\end{aligned}
$$

— L'aléatoire $\mathrm{I}$ - $\mathrm{S}_{j}$ est une indicatrice, par suite :

$$
\operatorname{var} \mathrm{I}-\mathrm{S}_{j}=\mathrm{E}\left(\mathrm{I}-\mathrm{S}_{j}\right)\left[\mathrm{I}-\mathrm{E}\left(\mathrm{I}-\mathrm{S}_{j}\right)\right]
$$

Pour le calcul de la covariance de $\mathrm{I}-\mathrm{S}_{j}$ et $\mathrm{X}_{j}^{\prime}$, nous utiliserons la relation :

$$
\mathrm{X}^{\prime}{ }_{j}\left(\mathrm{I}-\mathrm{S}_{j}\right)=\mathrm{o}
$$

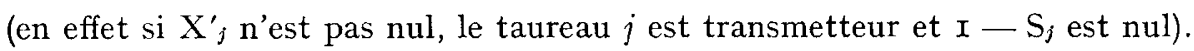

$$
\begin{aligned}
\operatorname{cov}\left(\mathrm{X}^{\prime}{ }_{j}, \mathrm{I}-\mathrm{S}_{j}{ }_{j}\right) & =\mathrm{E}\left[\mathrm{X}_{j}^{\prime}\left(\mathrm{I}-\mathrm{S}_{j}\right)\right]-\mathrm{E}\left(\mathrm{X}_{j}^{\prime}\right) \mathrm{E}\left(\mathrm{I}-\mathrm{S}_{j}\right)=-\mathrm{E}\left(\mathrm{X}_{j}^{\prime}\right) \mathrm{E}\left(\mathrm{I}-\mathrm{S}_{j}\right) \\
& =-m_{j} w^{\prime} \mathrm{G}\left(q, w^{\prime}\right) \mathrm{H}_{j}\left(q, w^{\prime}, \theta_{j}\right) /\left(\mathrm{I}-q^{2} w^{\prime}\right)
\end{aligned}
$$

De même :

$$
\operatorname{cov}\left(\mathrm{X}^{\prime \prime}{ }_{j}, \mathrm{I}-\mathrm{S}_{j}\right)=-f_{j} w^{\prime \prime} \mathrm{G}\left(q, w^{\prime}\right) \mathrm{H}_{j}\left(q, w^{\prime}, \theta_{j}\right) /\left(\mathrm{I}-q^{2} w^{\prime}\right)
$$

- L'indépendance des triplets $\left(\mathrm{X}^{\prime}{ }_{j}, \mathrm{X}^{\prime \prime}{ }_{j} . \mathrm{I}-\mathrm{S}_{j}\right)$ implique que les variances et covariances des aléatoires $\mathrm{X}_{0}^{\prime}, \mathrm{X}^{\prime \prime}{ }_{0}$ et $t-\mathrm{S}_{0}$ s'obtiennent en sommant les moments correspondants de $\mathrm{X}_{j}^{\prime}, \mathrm{X}^{\prime \prime}{ }_{j}$ et $\mathrm{I}-\mathrm{S}_{j}$ :

$$
\begin{aligned}
& =\mathrm{E}\left(\mathrm{X}_{0}^{\prime}\right)+q^{3}(\mathrm{I}-q)\left[\mathrm{I}-q w^{\prime}(2-q)\right] w^{\prime 2} \sum_{j=1}^{t} m^{2}{ }_{j} / 2\left(\mathrm{I}-q^{2} w^{\prime}\right)^{2} \\
& \operatorname{var} \mathrm{X}_{0}^{\prime} \\
& =\mathrm{E}\left(\mathrm{X}_{0}^{\prime \prime}\right)+q^{3}(\mathrm{I}-q)\left[\mathrm{I}-q w^{\prime}(2-q)\right] w^{\prime 2} \sum_{j=1}^{t} f_{j}^{2} / 2\left(\mathrm{I}-q^{2} w^{\prime}\right)^{2} \\
& \operatorname{var} \mathrm{X}^{\prime \prime}{ }_{0} \\
& \left.\operatorname{cov}\left(\mathrm{X}_{0}^{\prime}, \mathrm{X}_{0}^{\prime \prime}\right)=q^{3}(\mathrm{I}-q)\left[\mathrm{I}-q w^{\prime}\right)(2-q)\right] w^{\prime} w^{\prime \prime} \sum_{j=1}^{t} m_{j} t_{j} / 2\left(\mathrm{I}-q^{2} w^{\prime}\right)^{2} \\
& \operatorname{var}\left(t-\mathrm{S}_{0}\right) \quad=\mathrm{E}\left(t-\mathrm{S}_{0}\right)-\sum_{j=1}^{t}\left[\mathrm{H}_{j}\left(q, w^{\prime}, \theta_{j}\right)\right]^{2} /\left(\mathrm{I}-q^{2} w^{\prime}\right)^{2} \\
& \operatorname{cov}\left(\mathrm{X}_{0}^{\prime}, t-\mathrm{S}_{0}\right)=-q^{2} w^{\prime}\left(\mathrm{I}-q w^{\prime}\right) \sum_{j=1}^{t} m_{j} \mathrm{H}_{j}\left(q, w^{\prime}, \theta_{j}\right) /\left(\mathrm{I}-q^{2} w^{\prime}\right)^{2} \\
& \operatorname{cov}\left(\mathrm{X}_{0}^{\prime \prime}, t-\mathrm{S}_{0}\right)=-q^{2} w^{\prime \prime}\left(\mathrm{I}-q w^{\prime}\right) \sum_{j=1}^{t} f_{j} \mathrm{H}_{j}\left(q, w^{\prime}, \theta_{j}\right) /\left(\mathrm{I}-q^{2} w^{\prime}\right)^{2}
\end{aligned}
$$


III. - Calcul des derivées partielle; des fonctions a, b, c

Ce calcul classique ne présente aucune difficulté.

Les dérivées partielles des fonctions $a$ et $b$ se déduisent immédiatement de celles de la fonction $G$ :

$$
\begin{array}{lll}
\frac{\partial a}{\partial b}=m_{0} w^{\prime} \frac{\partial \mathrm{G}}{\partial q} & \frac{\partial a}{\partial w^{\prime}}=m_{0}\left(\mathrm{G}+w^{\prime} \frac{\partial \mathrm{G}}{\partial w^{\prime}}\right) & \frac{\partial a}{\partial w^{\prime \prime}}=0 \\
\frac{\partial b}{\partial q}=f_{0} w^{\prime \prime} \frac{\partial \mathrm{G}}{\partial q} & \frac{\partial b}{\partial w^{\prime}}=f_{0} w^{\prime \prime} \frac{\partial \mathrm{G}}{\partial w^{\prime}} & \frac{\partial b}{\partial w^{\prime \prime}}=f_{0} \mathrm{G}
\end{array}
$$

où

$$
\begin{aligned}
& \frac{\partial \mathrm{G}}{\partial q}=q\left(2-3 q w^{\prime}+q^{3} w^{\prime 2}\right) /\left(\mathrm{I}-q^{2} w^{\prime}\right)^{2} \\
& \frac{\partial \mathrm{G}}{\partial w^{\prime}}=-q^{3}(\mathrm{I}-q) /\left(\mathrm{I}-q^{2} w^{\prime}\right)^{2}
\end{aligned}
$$

Pour le calcul des dérivées partielles de la fonction $c$, il est commode de déterminer d'abord celles des fonctions $\mathrm{H}_{j}(j=\mathrm{I}, \ldots, t)$ considérées comme fonctions de $q, w^{\prime}, \theta_{j}$; les dérivées par rapport à $q, w^{\prime}, \theta_{j}$ seront écrites avec la notation $\delta$ pour ne pas être confondues avec les dérivées par rapport à $q, w^{\prime}, w^{\prime \prime}$ ( $\theta_{j}$ est en effet une fonction de ces trois variables).

$$
\begin{aligned}
& \frac{\delta \mathrm{H}_{j}}{\delta q}=-2\left[\mathrm{I}-q+q \exp \left(-\theta_{j}\right)\right]\left[\mathrm{I}-\exp \left(-\theta_{j}\right)\right]-2 q w^{\prime} \exp \left(-2 \theta_{j}\right) \\
& \frac{\delta \mathrm{H}_{j}}{\delta_{w}}=-q^{2} \exp \left(-2 \theta_{j}\right) \\
& \frac{\delta \mathrm{H}_{j}}{\delta \theta_{j}}=-2 q\left[\mathrm{I}-q+q \exp \left(-\theta_{j}\right)\right] \exp \left(-\theta_{j}\right)+2 q^{2} w^{\prime} \exp \left(-2 \theta_{j}\right)
\end{aligned}
$$

On en déduit les expressions suivantes :

$$
\begin{aligned}
\frac{\partial c}{\partial q} & =\frac{\mathrm{I}}{\mathrm{I}-q^{2} w^{\prime}} \sum_{j=1}^{t}\left[\frac{\delta \mathrm{H}_{j}}{\delta q}+\frac{m_{j} w^{\prime}+f_{j} w^{\prime \prime}}{2} \frac{\delta \mathrm{H}_{j}}{\delta \theta_{j}}\right]+\frac{2 q w^{\prime}}{\left(\mathrm{I}-q^{2} w^{\prime}\right)^{2}} \sum_{j=1}^{t} \mathrm{H}_{j} \\
\frac{\partial c}{\partial w^{\prime}} & =\frac{\mathrm{I}}{\mathrm{I}-q^{2} w^{\prime}} \sum_{j=1}^{t}\left[\frac{\delta \mathrm{H}_{j}}{\delta w^{\prime}}+\frac{q m_{j}}{2} \frac{\delta \mathrm{H}_{j}}{\delta \theta_{j}}\right]+\frac{q^{2}}{\left(\mathrm{I}-q^{2} w^{\prime}\right)^{2}} \sum_{j=1}^{t} \mathrm{H}_{j} \\
\frac{\partial c}{\partial w^{\prime \prime}} & =\frac{\mathrm{I}}{\mathrm{I}-q^{2} w^{\prime}} \sum_{j=1}^{t} \frac{q f_{j}}{2} \frac{\delta \mathrm{H}_{j}}{\delta \theta_{j}}
\end{aligned}
$$

IV. - Génotypes des taureaux reconnus transmetteurs

Connaissant le génotype du taureau $j$, les aléatoires $\mathrm{X}_{j}{ }_{j}$ et $\mathrm{X}^{\prime \prime}{ }_{j}$ sont des indépendantes et ont des lois de Poisson de paramètres connus.

La probabilité d'obtenir $x^{\prime}{ }_{j}$ mâles tarés et $x^{\prime \prime}{ }_{j}$ femelles tarées dans un lot de 
testage comprenant $m_{j}$ mâles et $f_{j}$ femelles est donc, si la somme $x_{j}{ }_{j}+x^{\prime \prime}{ }_{j}$ est non nulle (cas d'un transmetteur reconnu) :

pour un homozygote normal égale à 0 , pour un hétérozygote égale à :

$$
\begin{aligned}
& \Phi_{j}=\exp \left(-\frac{q m_{j} W^{\prime}}{2}\right) \frac{\mathrm{I}}{x_{j}^{\prime} !}\left(\frac{q m_{j} w^{\prime}}{2}\right)^{x_{j}} \times \exp \left(-\frac{q f_{j} w W^{\prime \prime}}{2}\right) \frac{\mathrm{I}}{x^{\prime \prime}{ }_{j} !}\left(\frac{q f_{j} w^{\prime \prime}}{2}\right)^{x^{\prime \prime}{ }_{j}} \\
& =\frac{\mathrm{I}}{x_{j}^{\prime} ! x^{\prime \prime}{ }_{j} !} \exp \left(-\theta_{j}\right)\left(\frac{q m_{j} w^{\prime}}{2}\right)^{x_{j}}\left(\frac{q f_{j} w^{\prime \prime}}{2}\right)^{x^{\prime \prime} j_{j}} / 2^{x_{j}+x^{\prime \prime}{ }_{j}}
\end{aligned}
$$

où

$$
\theta_{j}=q\left(m_{j} w w^{\prime}+f_{j} w^{\prime \prime}\right) / 2
$$

pour un homozygote récessif égale :

$$
\begin{aligned}
\Psi_{j} & =\exp \left(-q m_{j} w^{\prime}\right) \frac{\mathrm{I}}{x_{j}^{\prime} !}\left(q m_{j} w^{\prime}\right)^{x_{j}} \times \exp \left(-q f_{j} w^{\prime \prime}\right) \frac{\mathrm{I}}{x_{j}^{\prime \prime} !}\left(q f_{j} w^{\prime \prime}\right)^{x_{j}} \\
& =\frac{\mathrm{I}}{x_{j}^{\prime} ! x^{\prime \prime}{ }_{j} !} \exp \left(-2 \theta_{j}\right)\left(q m_{j} w^{\prime}\right)^{x_{j}}\left(q f_{j} w^{\prime \prime}\right)^{x_{j}}
\end{aligned}
$$

Les probabilités a priori de ces trois génotypes sont :

$$
\frac{(\mathrm{I}-q)^{2}}{\mathrm{I}-q^{2} w^{\prime}} \quad \frac{2 q(\mathrm{I}-q)}{\mathrm{I}-q^{2} w^{\prime}} \quad \frac{q^{2}\left(\mathrm{I}-w^{\prime}\right)}{\mathrm{I}-q^{2} w^{\prime}}
$$

Les probabilités a posteriori de ces génotypes (connaissant les résultats du testage) sont données par le théorème de Bayes. La probabilité que le taureau $j$ soit homozygote récessif est donc :

$$
\frac{\frac{q^{2}\left(\mathrm{I}-w^{\prime}\right)}{\mathrm{I}-q^{2} w^{\prime}} \Psi_{j}}{\frac{2 q(\mathrm{I}-q)}{\mathrm{I}-q^{2} w^{\prime}} \Psi_{j}+\frac{q^{2}\left(\mathrm{I}-w^{\prime}\right)}{\mathrm{I}-q^{2} w^{\prime}} \Psi_{j}}=\frac{2^{x^{\prime}{ }_{j}+x^{\prime \prime}{ }_{j}-1} q\left(\mathrm{I}-w^{\prime}\right) \exp \left(-\theta_{j}\right)}{\mathrm{I}-q+2^{x^{\prime} j+x^{\prime \prime} j^{-1}} q\left(\mathrm{I}-w^{\prime}\right) \exp \left(-\theta_{j}\right)}
$$

Pour les calculs numériques, les paramètres inconnus $q$, w' et $w^{\prime \prime}$ ont été remplacés par leurs estimations. 